Western University

Scholarship@Western

Robarts Imaging Publications

Robarts Research Institute

2014

\title{
Stationary wavelet transform for under-sampled MRI reconstruction.
}

Mohammad H Kayvanrad

A Jonathan McLeod

John S H Baxter

Charles A McKenzie

Terry M Peters

Follow this and additional works at: https://ir.lib.uwo.ca/robartspub

Part of the Bioimaging and Biomedical Optics Commons

Citation of this paper:

Kayvanrad, Mohammad H; McLeod, A Jonathan; Baxter, John S H; McKenzie, Charles A; and Peters, Terry M, "Stationary wavelet transform for under-sampled MRI reconstruction." (2014). Robarts Imaging Publications. 15.

https://ir.lib.uwo.ca/robartspub/15 


\title{
Stationary wavelet transform for under- sampled MRI reconstruction
}

Mohammad H. Kayvanrad ${ }^{* 1,2}$, A. Jonathan McLeod ${ }^{1,2}$, John S.H. Baxter ${ }^{1,2}$, Charles A. McKenzie ${ }^{1,2,3}$, Terry M. Peters ${ }^{1,2,3}$

\begin{abstract}
In addition to coil sensitivity data (parallel imaging), sparsity constraints are often used as an additional $l_{p}$-penalty for under-sampled MRI reconstruction (compressed sensing). Penalizing the traditional decimated wavelet transform (DWT) coefficients, however, results in visual pseudo-Gibbs artifacts, some of which are attributed to the lack of translation invariance of the wavelet basis. We show that these artifacts can be greatly reduced by penalizing the translation-invariant stationary wavelet transform (SWT) coefficients. This holds with various additional reconstruction constraints, including coil sensitivity profiles and total variation. Additionally, SWT reconstructions result in lower error values and faster convergence compared to DWT. These concepts are illustrated with extensive experiments on in vivo MRI data with particular emphasis on multiple-channel acquisitions.
\end{abstract}

Index terms: MRI reconstruction, Accelerated MR imaging, K-space undersampling, Sparse reconstruction, Compressed sensing, Parallel imaging

*Correspondence to: Mohammad Kayvanrad, Robarts Research Institute, 100 Perth Drive, London, ON, Canada.

Email: mkayvanrad@robarts.ca

${ }^{1}$ Robarts Research Institute, Western University, Canada

${ }^{2}$ Biomedical Engineering, Western University, Canada

${ }^{3}$ Medical biophysics, Western University, Canada 


\section{Introduction}

Cost considerations and patient comfort limit the total acceptable acquisition time in magnetic resonance imaging (MRI). On the other hand, it is necessary to acquire high-resolution images with high signal-to-noise ratio (SNR) for some applications. However, the SNR in MRI is proportional to the voxel volume and the square root of the acquisition time [1], which implies that high resolution and SNR are only achieved at the expense of long acquisition times. This in turn limits the number of pulse sequences that can be run in a clinical examination, which consequently limits the information that can be obtained. Therefore, there has been a strong motivation to reduce the acquisition time without compromising the resolution or the SNR of the MR images, since the introduction of this modality.

In addition to parallel imaging techniques [2]-[4], another approach to improving the trade-off between the acquisition time and the resolution is the acquisition of under-sampled k-space data and the use of the sparsity of the image in a transform domain, as an a priori reconstruction constraint, to interpolate the missing data. This approach is commonly referred to as compressed sensing or compressive sampling (CS) [5]-[8] and can be used in conjunction with parallel imaging [9].

Wavelet sparsity is commonly used as a reconstruction constraint in compressed sensing and sparse recovery applications. Conventionally, a weighted $l_{p}$-penalty on the decimated wavelet transform (DWT ${ }^{1}$ ) coefficients is used as a regularization term and the reconstruction problem is generally formulated as a constrained optimization- see [6]-[8], [10] and references therein.

It is well established in wavelet denoising that thresholding with the traditional DWT often results in pseudo-Gibbs artifacts, which are connected to the misalignment between the image features and the features of the wavelet basis [11]. For example, in Figure 1 a shift in the image results in misalignment between the image features and those of the wavelet basis after the shift (Figure 1(b)), which consequently results in a less sparse wavelet decomposition than that of the original image where the image features are deliberately chosen to match those of the wavelet basis (Figure 1(a)). Note that the shift does not change the energy of signal but after the shift the energy is spread over more [smaller] coefficients. A sparse decomposition is desirable in denoising as well as in sparse recovery applications since it allows the original features of the image to be distinguished from the noise or aliasing artifacts (and therefore enabling us to efficiently remove noise/artifacts, e.g., by thresholding) [7], [11]. This is pictorially shown in Figure 1 where thresholding results in visual reconstruction artifacts in Figure 1(b) due to the removal of a number of wavelet coefficients that fall below the threshold in the less

\footnotetext{
${ }^{1}$ DWT is also commonly used to abbreviate discrete wavelet transform. Since in this article we are essentially considering discrete cases only, any mention of the wavelet transform refers to the discrete wavelet transform (either decimated or undecimated). We use the abbreviation DWT to distinguish the decimated [discrete] wavelet transform from its undecimated version, i.e., the stationary wavelet transform (SWT).
} 
sparse representation. (Obviously, in this example one can avoid the artifacts by choosing a smaller threshold that maintains all the coefficients, but in practice a too small threshold fails to remove the noise/artifacts resulting in poor denoising/reconstruction. In this example the threshold is chosen to be $1 / 4^{\text {th }}$ of the largest coefficient, for the sake of illustration.) The effect of the choice of the threshold in practice is more thoroughly investigated in the Results section.
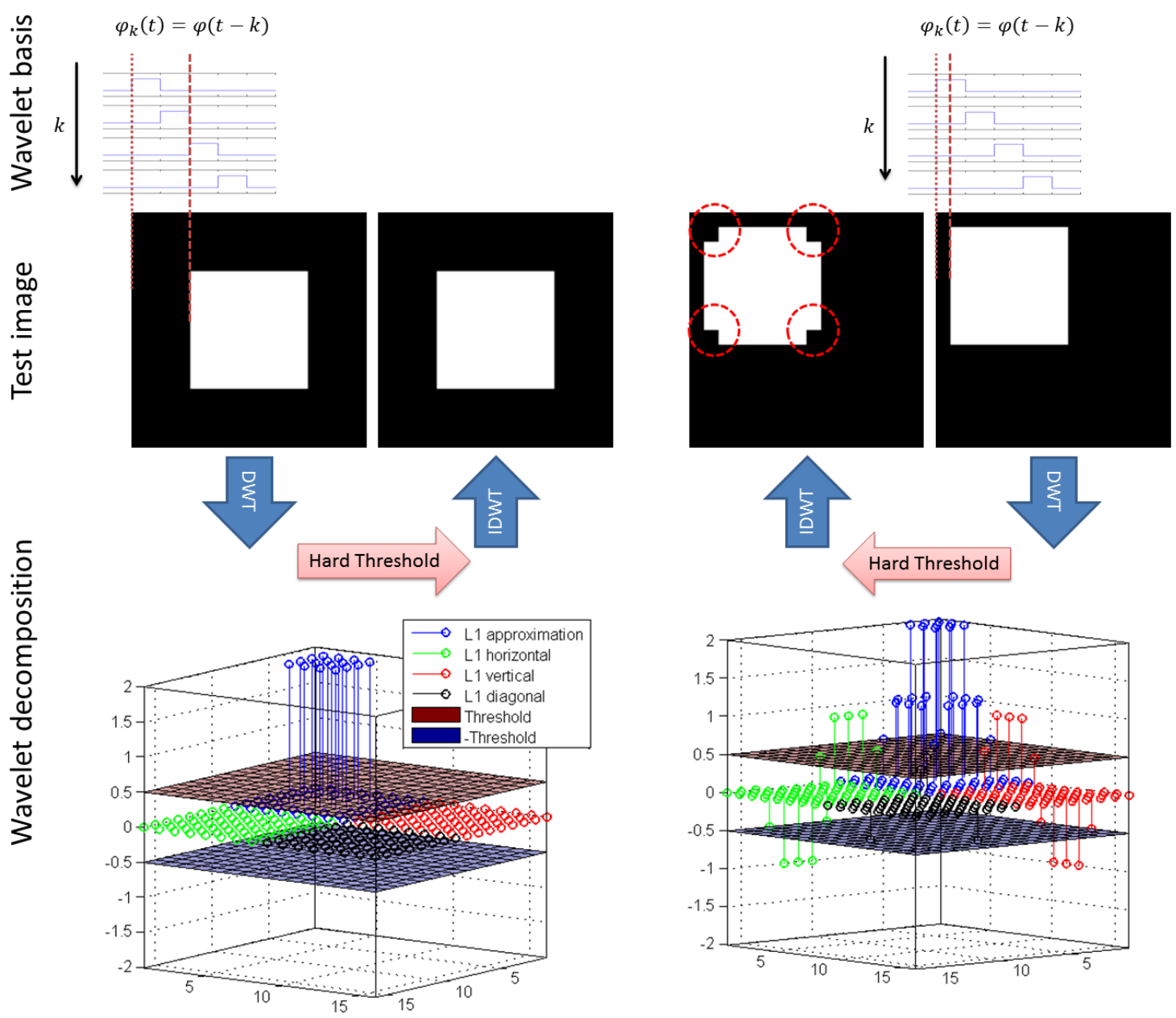

(a) Image features are aligned with those of the wavelet basis (dashed red line), resulting in a very sparse decomposition

(b) Image features are misaligned with those of the wavelet basis after the translation (dashed red line), resulting in a less sparse decomposition

Figure 1-Illustration of the lack of translation invariance of DWT and the resulting thresholding artifacts: A simple test image- an 8x8 square in the middle of a 16x16 black background (a) and a shifted version of it (b) are decomposed with the Haar wavelet to 1 level. The original image is deliberately chosen to align with the wavelet basis, resulting in a very sparse decomposition. The shift, however, results in a misalignment between the image features and those of the wavelet basis functions, which, consequently, results in noticeable loss of the sparsity of the decomposition. In each case, the decomposition coefficients are hard thresholded and a wavelet reconstruction (IDWT) is performed on the thresholded coefficients. Dashed circles highlight the reconstruction artifacts. 
One could possibly avoid the misalignment between the image features and those of the wavelet basis by shifting the image or the basis functions to make them aligned. However, this requires a priori knowledge of the best aligning shift. Furthermore, when the image contains several discontinuities, there may not be a single shift that works for all the discontinuities- the best shift for one may be the worst for the other. Consequently, Coifman and Donoho proposed the idea of "translationinvariant denoising," i.e., average[shift-denoise-unshift] for several (or all possible) shifts [11]. This, in practice, is often achieved by stationary wavelet transform (SWT) thresholding, which provides a translation-invariant basis [12], [13]. For the sake of completeness, a brief description of SWT based on [12] follows. For simplicity, we consider the 1D discrete case only- extension to 2D is straight forward.

DWT decomposition of a signal $x(t)$ results in the scaling (approximation) and wavelet (detail) coefficients:

$$
\begin{aligned}
& c_{j}^{k}=\left\langle x(t), 2^{-j / 2} \varphi\left(\frac{t}{2^{j}}-k\right)\right\rangle \\
& d_{j}^{k}=\left\langle x(t), 2^{-j / 2} \psi\left(\frac{t}{2^{j}}-k\right)\right\rangle
\end{aligned}
$$

where $\varphi(t)$, and $\psi(t)$, are the scaling and wavelet functions, respectively, and $j$ and $k$ amount to the scaling and translation of the wavelet basis, respectively.

For SWT, a redundant decomposition can be obtained as,

$$
\begin{aligned}
& \tilde{c}_{2^{j}}^{2^{j} k+p}=\left\langle x(t), 2^{-j / 2} \varphi\left(\frac{t-p}{2^{j}}-k\right)\right\rangle \\
& \tilde{d}_{2^{j}}^{2^{j} k+p}=\left\langle x(t), 2^{-j / 2} \psi\left(\frac{t-p}{2^{j}}-k\right)\right\rangle
\end{aligned}
$$

where $p \in\left\{0, \cdots, 2^{j}-1\right\}$ allows for all the possible shifts in a discrete setting.

For decomposition to $j_{m}$ levels, $2^{j_{m}}$ different orthogonal bases can be generated. The different possible choices can be illustrated by a binary tree in the form of Figure 2. Each node in this tree is indexed by parameters $(j, p)$, to which the set of coefficients $\left\{\tilde{c}_{2^{j}}^{2^{j} k+p}\right\}_{k \in \mathbb{Z}}$ is associated. Each path from the root of the tree to a leaf corresponds to the set of functions $\left\{2^{-j / 2} \psi\left[\left(t-p_{j}\right) / 2^{j}-k\right], k \in \mathbb{Z}, 1 \leq j \leq j_{m}\right\} \cup$ $\left\{2^{-j_{m} / 2} \varphi\left[\left(t-p_{j_{m}}\right) / 2^{j_{m}}-k\right], k \in \mathbb{Z}\right\}$, which forms an orthogonal wavelet basis, resulting in a standard wavelet reconstruction. The inverse SWT is often defined as the average of all the $2^{j_{m}}$ different reconstructions obtained in this manner. 


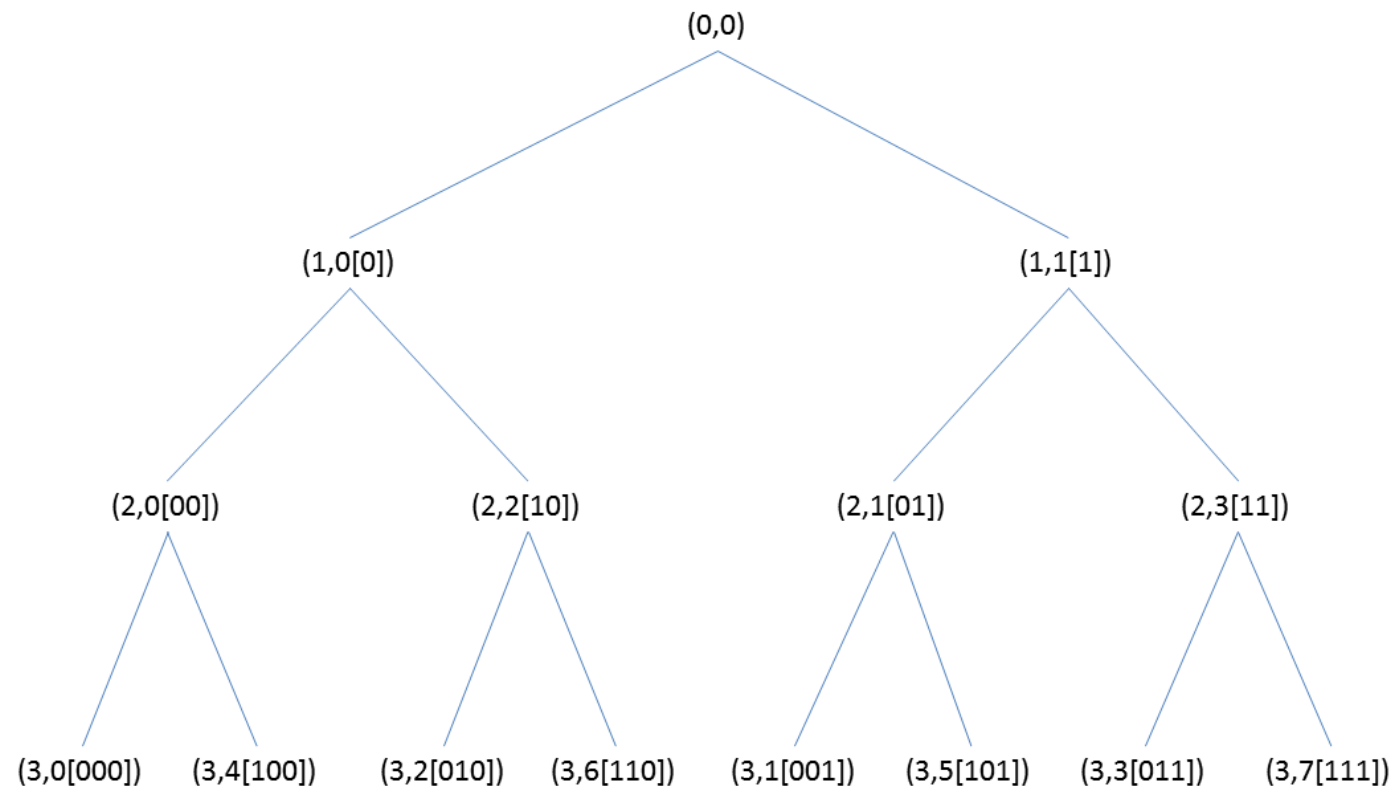

Figure 2- Shift-localization tree for a three level stationary wavelet transform (SWT) decomposition. Each node is indexed by parameters $(j, p)$, where $j$ is the decomposition level and $p$ is the shift. For clarity, the binary representation of $p$ is shown in brackets.

While SWT is predominantly used in wavelet denoising, to the best of our knowledge, the use of SWT in compressed sensing and sparse recovery applications, particularly in under-sampled MRI reconstruction, has not been explored before. The key idea here is that the $l_{p}$-penalty on the DWT coefficients essentially results in the same sort of artifacts described above, which can be avoided or reduced by penalizing the SWT coefficients.

The intent of this article is to call attention to the benefits of the use of SWT in place of DWT for compressed sensing and sparse recovery, with particular focus on MRI reconstruction from under-sampled k-space data. Although use of SWT for such applications may seem counter intuitive, since it is a redundant transform, we show that significant improvement in reconstruction quality is achieved by replacing the $l_{p}$-penalty on the DWT coefficients with one on the SWT coefficients. This holds even with additional constraints, including total variation (TV) penalties or coil sensitivity constraints when compressed sensing is combined with parallel imaging.

Furthermore, a few authors have recently reported the use of DWT with random shifts (DWTRS) [14] to address the DWT translation variance problem for compressed sensing and sparse recovery applications [15], [16]. In this article, use of random shifts with decimated wavelet transform is also considered in 
comparison with the conventional decimated wavelet transform as well as its undecimated version, i.e., SWT.

In should be noted that several extensions to the wavelet transform, including the dual-tree wavelet transform [17], contourlets [18], and curvelets [19], have been used for compressed sensing applications, including MRI reconstruction, to address specific limitations of the regular decimated wavelet transform [20]-[23]. However, a comprehensive study/comparison of all these transforms is beyond the scope of this work. This work compares variations of the standard discrete wavelet transform, i.e., in its commonly-used decimated form (DWT), with random shifts (DWTRS), and its undecimated version (SWT). Note that unlike other extensions, such as dual-tree wavelets, curvelets, and contourlets, these transforms are directly based on the standard wavelet transform, e.g., they are all based on the same wavelet and scaling functions and only differ in terms of shift and/or decimation. As noted, our main intention is to call attention to the advantages of the redundant shift-invariant version of the standard discrete wavelet transform, i.e., SWT, in comparison with its decimated versions, i.e., DWT, and DWTRS, which is the most widely studied sparsifying transform and most commonly used in practice as the state-of-the-art reconstruction by several authors (see for example [16], [24], [25]).

In addition to reduced visual artifacts, SWT results in significantly lower reconstruction error as well as faster convergence. Furthermore, despite its redundancy, it can be computed rapidly- in $n \log (n)$ time [11].

All these concepts are illustrated by extensive experiments with different reconstruction techniques, all of which are reproducible using the supplementary code provided with this paper or the code supplied by the authors cited in this paper.

\section{Stationary wavelet transform sparse recovery}

In the discussion that follows we perform a point spread function (PSF) 2 analysis to demonstrate the performance of SWT thresholding in comparison with DWT thresholding for removing under-sampling aliasing artifacts. A computational experiment with the Shepp-Logan phantom is also presented to illustrate the visual artifacts in the DWT reconstruction compared with those in the SWT reconstruction.

Let $f(m, n)=\delta(m, n)$ be an impulse input in the spatial domain, where $\delta(m, n)=$ $\left\{\begin{array}{cc}1 & m=0, n=0 \\ 0 & \text { otherwise }\end{array}\right.$. Transforming $f$ to the Fourier domain, under-sampling, and transforming back to the spatial domain results in the k-space under-sampling PSF, $f_{u}$ (Figure 3(a)):

\footnotetext{
${ }^{2}$ A linear shift-invariant imaging system can be completely described in terms of its point spread function (PSF). Although thresholding is a non-linear operation, we still use the PSF for illustration/comparison of the artifacts.
} 


$$
f_{u}=\mathcal{F}^{-1} U_{F} \mathcal{F} f
$$

where $U_{F}$ is the Fourier (k-space) under-sampling operation and $\mathcal{F}$ is the Fourier transform.

Now consider the wavelet decomposition of $f_{u}$ using DWT and SWT: $C_{D W T}=$ $\psi_{D W T} f_{u}$, and $C_{S W T}=\psi_{S W T} f_{u}$, where $\psi_{D W T}$ and $\psi_{S W T}$ are the decimated and stationary wavelet transforms, and $C_{D W T}$ and $C_{S W T}$ are the corresponding wavelet decomposition coefficients. Assume a thresholding operation, $\gamma$, acting on the decomposition coefficients: $\tilde{C}_{D W T}=\gamma\left(C_{D W T}\right)$, and $\tilde{C}_{S W T}=\gamma\left(C_{S W T}\right)$. The corresponding PSFs are computed by wavelet reconstruction of the thresholded coefficients: $\tilde{f}_{D W T}=\psi_{D W T}^{*} \tilde{C}_{D W T}$, and $\tilde{f}_{S W T}=\psi_{S W T}^{*} \tilde{C}_{S W T}$. Figure 3(b, c) show the DWT and SWT soft thresholding PSFs, respectively. The same threshold, chosen using the Birgé-Massart strategy [26], is used with both DWT and SWT. (The choice of the threshold and its effect on the reconstruction is more thoroughly investigated in the Results section.) Note that with the assumption of under-sampling in the phaseencode $\left(k_{y}\right)$ direction only, the point spread functions can be sufficiently illustrated with 1D plots. An example of the reduction of the artifacts by SWT thresholding compared to DWT thresholding is highlighted. Several such reductions can be easily identified on the PSFs. As illustrated in Figure 3, SWT thresholding results in noticeably fewer artifacts than the corresponding DWT thresholding. 
(a) Under-sampling PSF

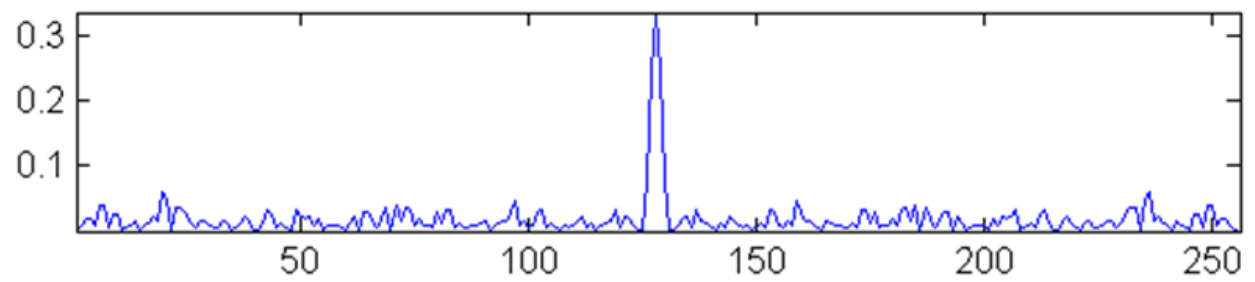

(b) DWT PSF

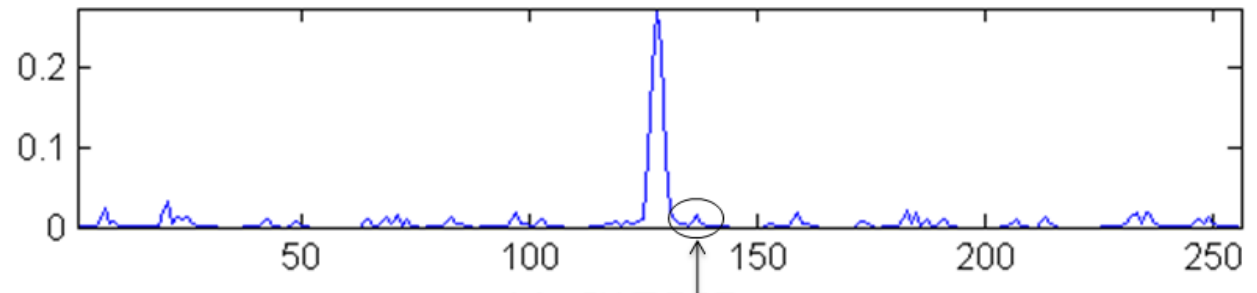

(c) SWTPSF

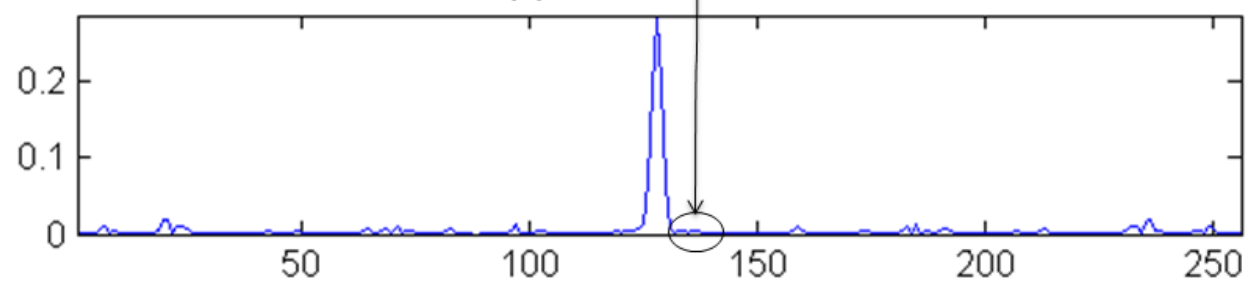

Figure 3- Point spread functions (PSF) resulting from k-space under-sampling (a) followed by the application of DWT and SWT soft thresholding (b and c, respectively). An example of reduced artifacts by SWT thresholding in comparison with DWT thresholding is highlighted.

Since the aliasing artifacts are incoherent ${ }^{3}$, the signal-to-alias ratio, defined as the energy of the signal (i.e., the peak in this case) to the energy of the alias (i.e., the side-lobes in this case) of the PSFs, provides a quantitative means of comparing these PSFs with each other and with the under-sampling PSF (Table 1). The higher signal-to-alias ratio achieved by SWT thresholding also indicates less aliasing interference.

\begin{tabular}{lr}
\hline & Signal-to-alias ratio \\
\hline Under-sampling PSF & 0.506 \\
DWT thresholding PSF & 0.647 \\
SWT thresholding PSF & 0.912 \\
\hline
\end{tabular}

\footnotetext{
${ }^{3}$ In compressed sensing, it is desired to have incoherent (noise-like) under-sampling artifacts so that they can be distinguished from the original signal/image features in the sparse domain [7]. The incoherence is often achieved through random under-sampling.
} 
Table 1- Signal-to-alias ratios corresponding to the point spread functions (PSF) in Figure 3.

In order to illustrate the nature of the artifacts associated with the DWT reconstruction, consider the computational experiment of reconstruction of the Shepp-Logan phantom (Figure 4(a)) from under-sampled frequency domain data. For the sake of illustration, and since Cartesian sampling is by far the most common way of acquiring k-space data in MRI, we assume Cartesian under-sampling in the $y$ direction (corresponding to under-sampling in the phase-encode direction in an MRI application).

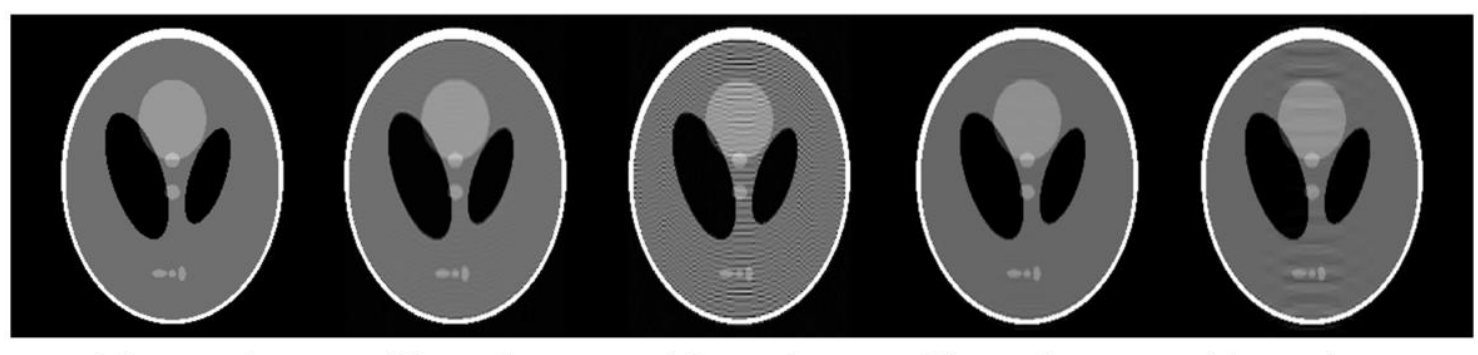

(a) Original

(b) SWT $l_{1}$

(c) DWT $l_{1}$

(d) SWT $l_{1}+\mathrm{TV}$

(e) DWT $l_{1}+$ TV

Figure 4- Reconstruction of the Shepp-Logan phantom from Cartesian under-sampled frequency data by DWT/SWT- $l_{1}(+\mathrm{TV})$ penalized optimization.

Figure 4(b,c) show the reconstruction of the under-sampled frequency domain data based on an $l_{1}$ penalized optimization, i.e., $\min _{f^{\star}}\left\|\psi f^{\star}\right\|_{l_{1}}$ s.t. $\left\|U_{F} \mathcal{F} f^{\star}-F_{u}\right\|_{l_{2}}<\varepsilon$, where the reconstruction in Figure $4(\mathrm{c})$ is achieved when $\psi=\psi_{D W T}$ is a decimated wavelet transform and that of Figure $4(\mathrm{~b})$ is achieved when $\psi=\psi_{S W T}$ is the corresponding stationary wavelet transform. Here $f^{\star}$ denotes the reconstructed image, $\mathcal{F}$ the Fourier transform, $U_{F}$ the under-sampling operation in the frequency domain, and $F_{u}$ the acquired frequency data. As shown in Figure $4(b, c)$, most of the artifacts present in the DWT reconstruction are absent in the SWT reconstruction.

Furthermore, several authors have reported that it is often useful to include an additional total variation (TV) penalty in the reconstruction [7], [27]. Since all the previous works were based on penalizing the DWT coefficients, the TV term was needed to alleviate the associated artifacts. However, as illustrated by the above example, penalizing the SWT coefficients may reduce the need for the additional TV penalty. Nevertheless, as illustrated in Figure 4(d,e) SWT is preferred over DWT with an additional TV penalty, i.e., $\min _{f^{\star}}\left\|\psi f^{\star}\right\|_{l_{1}}+\alpha T V\left(f^{\star}\right)$ s.t. $\left\|U_{F} \mathcal{F} f^{\star}-F_{u}\right\|_{l_{2}}<\varepsilon$, also. It should be noted that the Shepp-Logan phantom heavily favors a TV penalty (perfect reconstruction has been demonstrated for the Shepp-Logan phantom with a TV penalty with radial under-sampling [5]). Such drastic improvement with an additional TV term may not be observed with real MR images though. 


\section{Methods}

Single channel spoiled gradient recalled (SPGR) data of a healthy volunteer were acquired at 3T (matrix: $256 \times 256$, resolution $=0.86 \mathrm{~mm}$ isotropic, slice thickness $=$ $1 \mathrm{~mm}, \mathrm{TE} / \mathrm{TR}=4.1 \mathrm{~ms} / 8.9 \mathrm{~ms}, \mathrm{BW}= \pm 19.23 \mathrm{kHz}$, flip angle $=18^{\circ}, \mathrm{NEX}=1$ ). Human data used in this work were acquired using a protocol approved by the University of Western Ontario Office Of Research Ethics.

k-space data were retrospectively under-sampled in the phase-encode direction and the under-sampled data were reconstructed by SWT $l_{1}+$ TV penalized and DWT $l_{1}+$ TV penalized optimization, i.e.,

$$
\min _{f^{\star}}\left\|\psi f^{\star}\right\|_{l_{1}}+\alpha T V\left(f^{\star}\right) \text { s.t. }\left\|U_{F} \mathcal{F} f^{\star}-F_{u}\right\|_{l_{2}}<\varepsilon \text { (6) }
$$

with $\psi=\psi_{S W T}$ and $\psi=\psi_{D W T}$, respectively. The optimization was performed using the code provided by Lustig et al for [7].

In practice it is expected to achieve the best under-sampled reconstruction performance by the combined application of compressed sensing and parallel imaging.

Iterative thresholding reconstruction [10] can be modified to directly incorporate the coil sensitivity profiles. The multiple-coil iterative thresholding reconstruction algorithm is shown in Table 2 .

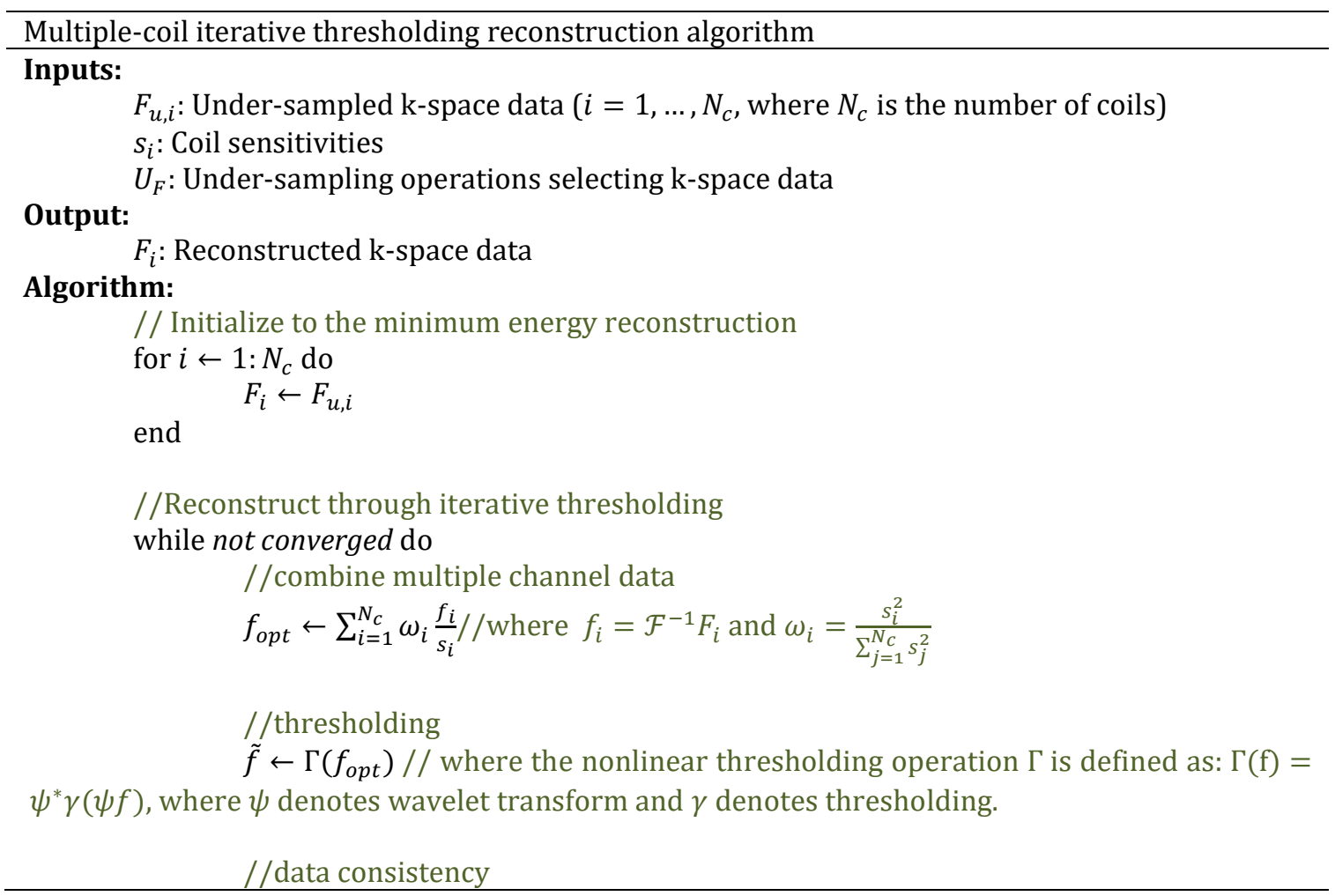




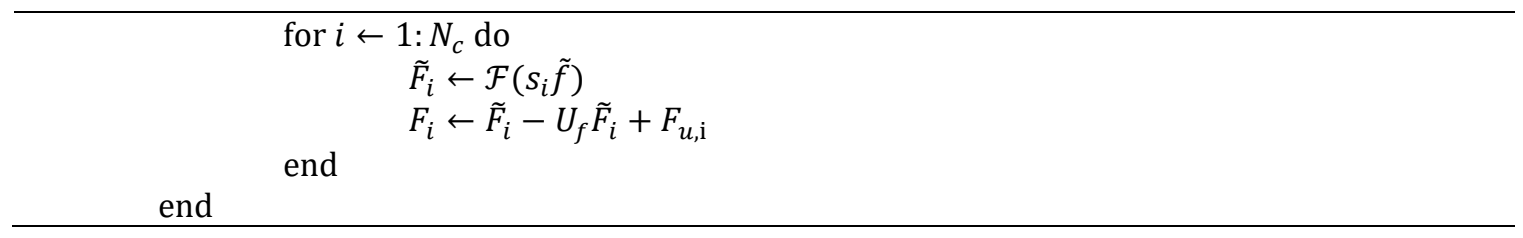

Table 2- Multiple-coil iterative thresholding reconstruction algorithm. $\mathcal{F}$ and $\Gamma$ denote the Fourier transform and wavelet thresholding operations, respectively.

A flow chart of the multiple-coil reconstruction procedure is shown in Figure 5. In Step 1 the combined-channels image is modulated by the coil sensitivity profile of each channel in order to make the combined-channels estimate consistent with the coil data before the data projection in Step 2, which enforces the data consistency constraint for each channel by projecting the current estimate onto the corresponding coil data [28]. In Step 3 data from multiple channels are combined to obtain a combined-channels estimate image. If coil sensitivities are explicitly available, an optimal combination has been shown by Roemer to be [29]:

$$
f_{\text {opt }}=\sum_{i=1}^{N_{c}} \omega_{i} \frac{f_{i}}{s_{i}} \quad \text { where } \omega_{i}=\frac{s_{i}^{2}}{\sum_{j=1}^{N_{c}} s_{j}^{2}}
$$

where $f_{i}$ is the image from the $i$ th coil and $s_{i}$ is the corresponding coil sensitivity profile. In practice, the coil sensitivities are commonly extracted from fully-sampled low-resolution reference data ${ }^{4}$, which can be acquired prior to the main scan (precalibration) or integral to the main scan (auto-calibration) by fully sampling a region over the center of k-space[30]. We use the latter approach to estimate the coil sensitivities.

\footnotetext{
${ }^{4}$ A simple approach to computing the sensitivity profiles from reference data, which is commonly used in practice, is to divide each native coil image by the sum of squares [30].
} 


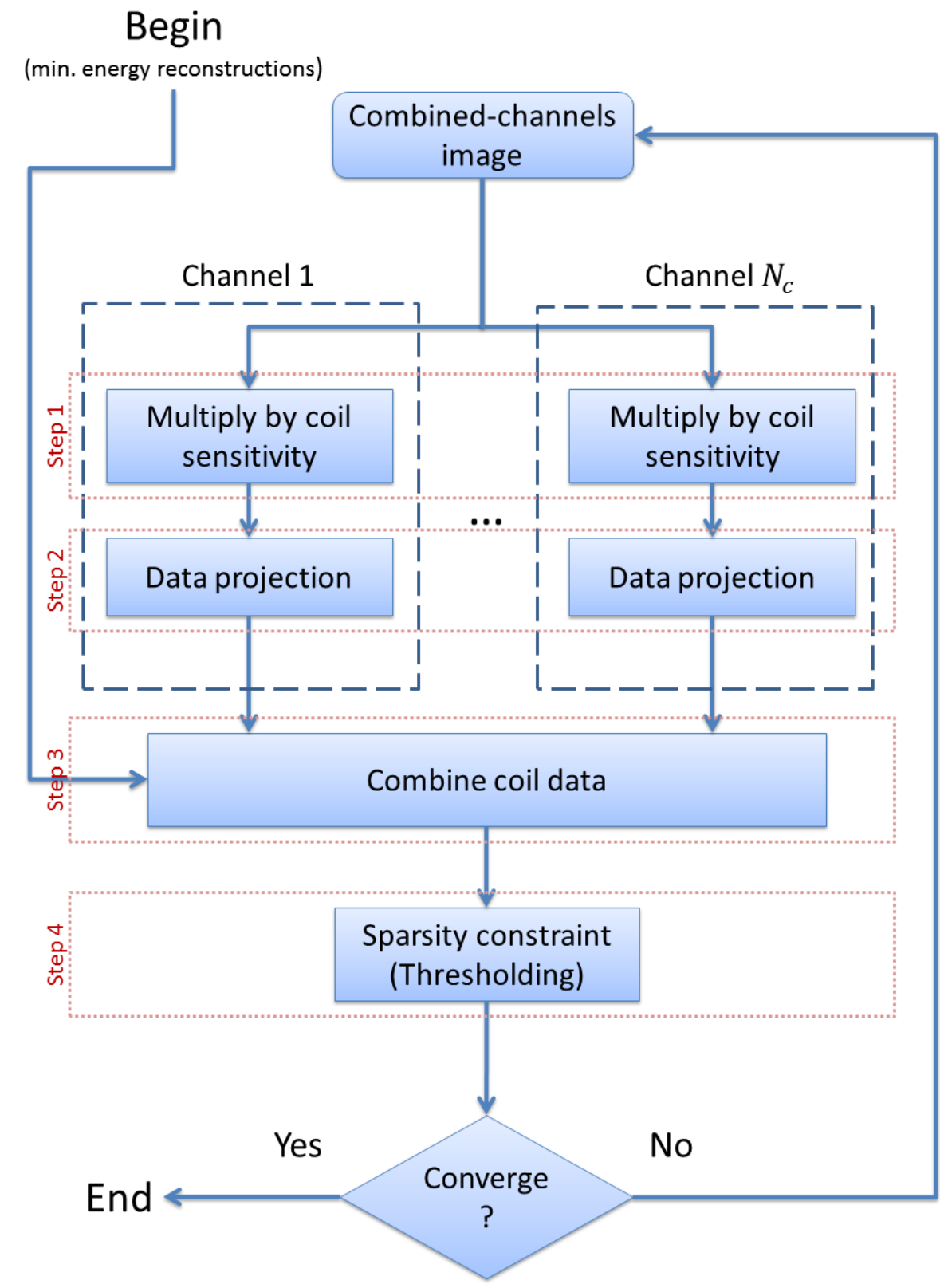

Figure 5- Flowchart of the multiple-coil iterative thresholding reconstruction algorithm

Finally, in Step 4, the sparsity constraint is enforced through a thresholding operation.

The approach to incorporating coil sensitivity data in the reconstruction algorithm is similar to the POCS-based parallel imaging reconstruction algorithm described by Samsonov et al [28]. Note that this approach does not impose any constraint on the k-space under-sampling pattern. 
The multiple-coil reconstruction algorithm amounts to thresholded Landweber iterations, which has been proved to converge with soft thresholding by Daubechies [10]. Nevertheless, we also experimentally investigate reconstruction by hard thresholding to show the effectiveness of SWT with both soft and hard thresholding 5 .

Brain images of a healthy volunteer were acquired at 3T using a 32-channel head coil with a 2D fast spin echo (FSE) pulse sequence (matrix: 256x256, resolution = $1 \mathrm{~mm}$, slice thickness $=2 \mathrm{~mm}, \mathrm{TR} / \mathrm{TE}=3600 \mathrm{~ms} / 80 \mathrm{~ms}, \mathrm{ETL}=15, \mathrm{BW}= \pm 15.63 \mathrm{kHz}$, NEX $=1$ ). A portion of $k$-space at the center was fully sampled to generate the lowresolution auto-calibration data with the rest of k-space under-sampled with variable density in the phase encode direction. K-space data were then reconstructed by the multiple-coil iterative thresholding reconstruction algorithm with SWT $\left(\Gamma=\Gamma_{S W T}\right)$ and DWT $\left(\Gamma=\Gamma_{S W T}\right)$. The experiments were repeated for a range of under-sampling factors from 2 to 6.

While the under-sampling pattern is chosen at random to achieve incoherent undersampling, it is known that the performance of compressed sensing reconstructions is affected by the choice of the under-sampling pattern. In order to avoid the possibility of randomly picking a "bad" under-sampling pattern, some authors have proposed to generate a number of under-sampling patterns and picking one that maximizes the incoherence [7], while in practice some other authors used pilot studies to pick the best under-sampling pattern for the data [25]. In order to consider the dependence on the random under-sampling pattern in general in our comparisons, repeated trials with 15 sets of random under-sampling patterns, generated independently, were carried out for each under-sampling factor, based on which the statistical significance of findings was evaluated using paired t-tests.

It should be noted that with 2D under-sampling for 3D acquisitions a reasonable under-sampling pattern can be generated using a Poisson disk sampling scheme [9]. We take the latter approach for 2D under-sampling of 3D sequences as described later in this section.

To further examine the applicability of SWT to multiple-coil reconstructions, the aforementioned under-sampled data were also reconstructed by the SPIRiT

$$
\begin{aligned}
& \text { 5 Soft thresholding is defined as: } \gamma_{\mu}(x)=\left\{\begin{array}{cc}
x+\frac{\mu}{2} & \text { if } \mathrm{x} \leq-\frac{\mu}{2} \\
0 & \text { if }|x|<\frac{\mu}{2} \\
x-\frac{\mu}{2} & \text { if } x \geq \frac{\mu}{2}
\end{array} \text {, where } \mu\right. \text { is the threshold. Similarly, } \\
& \text { hard thresholding is defines as: } \gamma_{\mu}(x)=\left\{\begin{array}{cc}
x & \text { if } \mathrm{x} \leq-\frac{\mu}{2} \\
0 & \text { if }|x|<\frac{\mu}{2} \\
x & \text { if } x \geq \frac{\mu}{2}
\end{array}\right.
\end{aligned}
$$


reconstruction method described in [9], where the reconstruction problem is formulated as an optimization with calibration and wavelet $l_{1}$ penalties, subject to consistency with the acquired data:

$$
\min _{f^{\star}}\left\|\psi f^{\star}\right\|_{l_{1}}+\lambda\left\|(G-I) \mathcal{F} f^{\star}\right\|_{l_{2}} \text { s.t. }\left\|U_{F} \mathcal{F} f^{\star}-F_{u}\right\|_{l_{2}}<\varepsilon
$$

Where $f^{\star}$ is now the solution consisting of every and each individual coil. Similarly $F_{u}$ consists of under-sampled data acquisition for all coils. $G$ is the SPIRiT calibration operator and $I$ is the unitary matrix. The difference between the SPIRiT calibration operator and that of the traditional GRAPPA [3] is that in SPIRiT the calibration operator is a "full" kernel independent of the under-sampling pattern, which is the same for all k-space positions. For more details refer to [9]. Reconstruction was performed with SWT, DWT, and DWT with random shifts (DWTRS), using the code provided by the authors.

Reconstruction quality was quantitatively measured in terms of the normalized root mean square error (NRMSE) with respect to the fully-sampled data. The statistical significance of the findings was evaluated by paired comparisons of the NRMSE values based on paired t-tests under the null hypothesis that the mean NRMSE of the DWT reconstruction in each pair is smaller than or equal to that of SWT. Since several such t-tests were performed, the comparisons were corrected by the Bonferroni correction with a significance level of $\alpha=0.05$.

Furthermore, as noted previously, it has been shown that reasonable 2D undersampling can be achieved using a Poisson disk sampling scheme for 3D acquisitions. 3D SPGR data were acquired at 3T using a 32-channel head coil (matrix: $256 \times 256 \times 160$, resolution $=1 \mathrm{~mm}$ isotropic, TE $/ \mathrm{TR}=3.71 \mathrm{~ms} / 8.36 \mathrm{~ms}$, flip angle $=$ $18^{\circ}, \mathrm{BW}= \pm 19.23 \mathrm{kHz}, \mathrm{NEX}=1$ ). K-space data were retrospectively under-sampled in the phase-encode directions using a Poisson disk under-sampling scheme, and reconstructed by SPIRiT with DWT, DWTRS, and SWT. 


\section{Results}

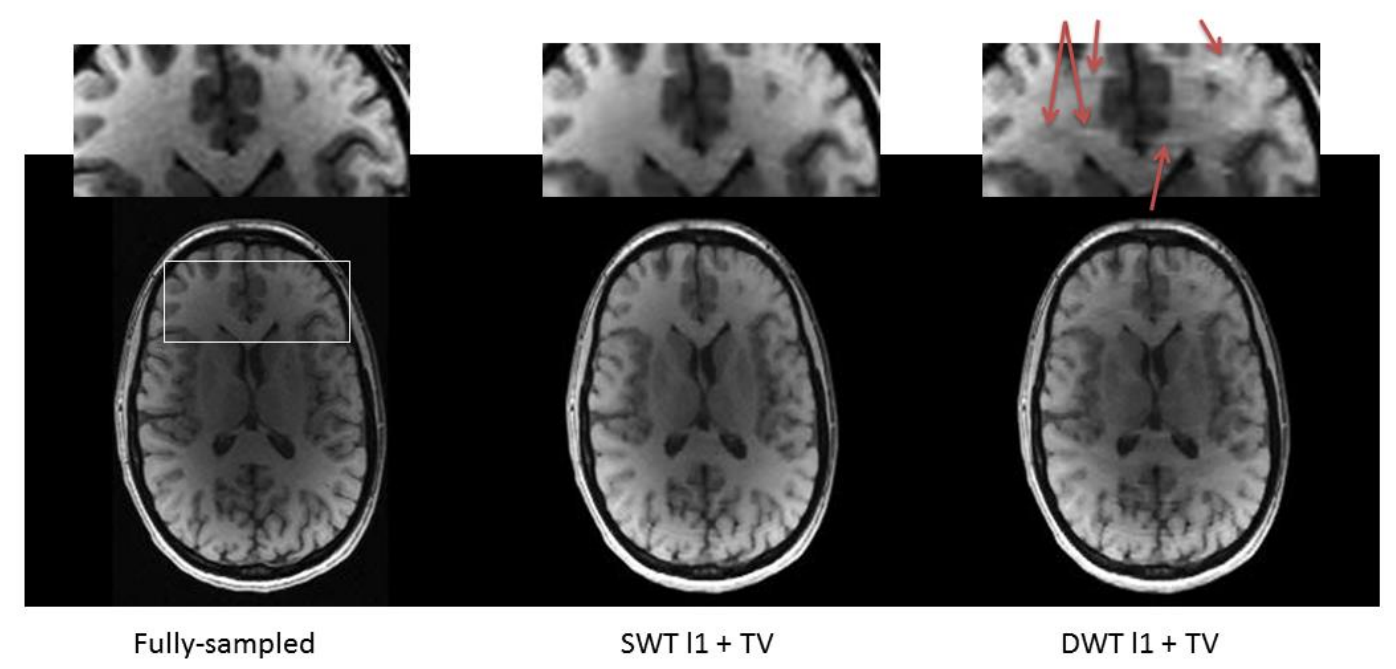

Figure 6- Reconstruction of under-sampled SGPR data (under-sampling factor 3 ) by $l_{1}+$ TV penalized optimization. The arrows point examples of the artifacts present in the DWT reconstruction that are absent in the corresponding SWT reconstruction.

Figure 6 shows the reconstruction of the under-sampled SPGR data by SWT/DWT $l_{1}$ $+\mathrm{TV}$ penalized optimization. The choice of the regularization parameter $(\alpha$ in equation 6) generally affects the reconstruction performance. In order to avoid the possibility of giving SWT any advantage over DWT by a particular choice of $\alpha$, a value optimized for the DWT reconstruction (suggested by Lustig et al in their code) was used for both reconstructions. The dependence of the SWT/DWT reconstructions on the regularization parameter is more thoroughly investigated in the next experiments. The images clearly illustrate DWT reconstruction artifacts (even with an additional TV penalty) that are absent in the SWT reconstruction.

Figure 7 shows the results of the repeated trials for the reconstruction of the undersampled 32-channel FSE data by the multiple-coil iterative thresholding algorithm. Clearly, the SWT reconstructions resulted in lower mean error values than the corresponding DWT reconstructions. The mean and its $95 \%$ confidence interval of the paired NRMSE differences of the SWT and DWT reconstructions, i.e., NRMSEsWTNRMSEdwT, corresponding to the paired t-tests, are shown in Table 3 for both hard and soft thresholding. Negative NRMSE difference means with confidence intervals that do not include zero indicate that SWT resulted in lower reconstruction errors than DWT for both soft and hard thresholding. Furthermore, after the Bonferroni correction all the findings were significant. 


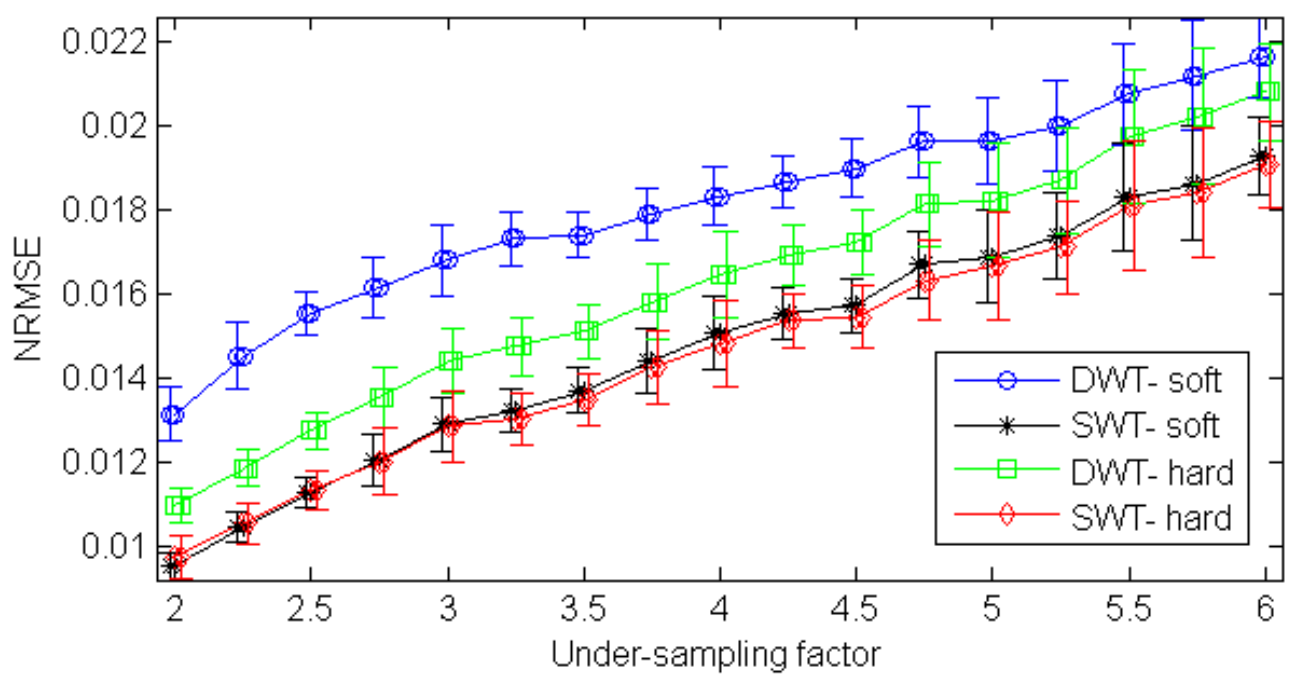

Figure 7- Mean NRMSE and the corresponding error bars of one standard deviation for the reconstruction of the under-sampled 32-channel FSE data by the multiple-coil iterative thresholding algorithm.

NRMSEswT - NRMSE ${ }_{\text {DWT }}\left(\mathrm{x} 10^{-3}\right)$

\begin{tabular}{lccccc}
\hline U.F. & 2 & 3 & 4 & 5 & 6 \\
\hline Soft & $-3.6(37 \%) \pm 0.16$ & $-3.9(30 \%) \pm 0.22$ & $-3.2(22 \%) \pm 0.18$ & $-2.7(16 \%) \pm 0.17$ & $-2.4(12 \%) \pm 0.18$ \\
Hard & $-1.2(13 \%) \pm 0.12$ & $-1.6(12 \%) \pm 0.12$ & $-1.6(11 \%) \pm 0.24$ & $-1.5(9 \%) \pm 0.18$ & $-1.7(9 \%) \pm 0.16$ \\
\hline
\end{tabular}

Table 3- Mean and its 95\% confidence interval of the paired NRMSE differences (i.e., NRMSE of the DWT reconstruction subtracted from that of the corresponding SWT reconstruction) for the reconstruction of the 32-channel FSE data by the multiple-coil iterative thresholding algorithm with soft and hard thresholding for different under-sampling factors (U.F.). The numbers in brackets show the percentage of mean improvement with SWT over DWT. All findings in this table are statistically significant after the Bonferroni correction. 


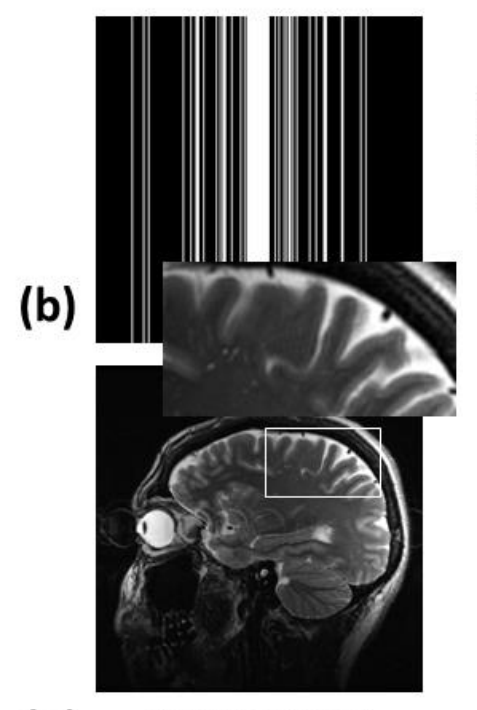

(a) Fully-sampled

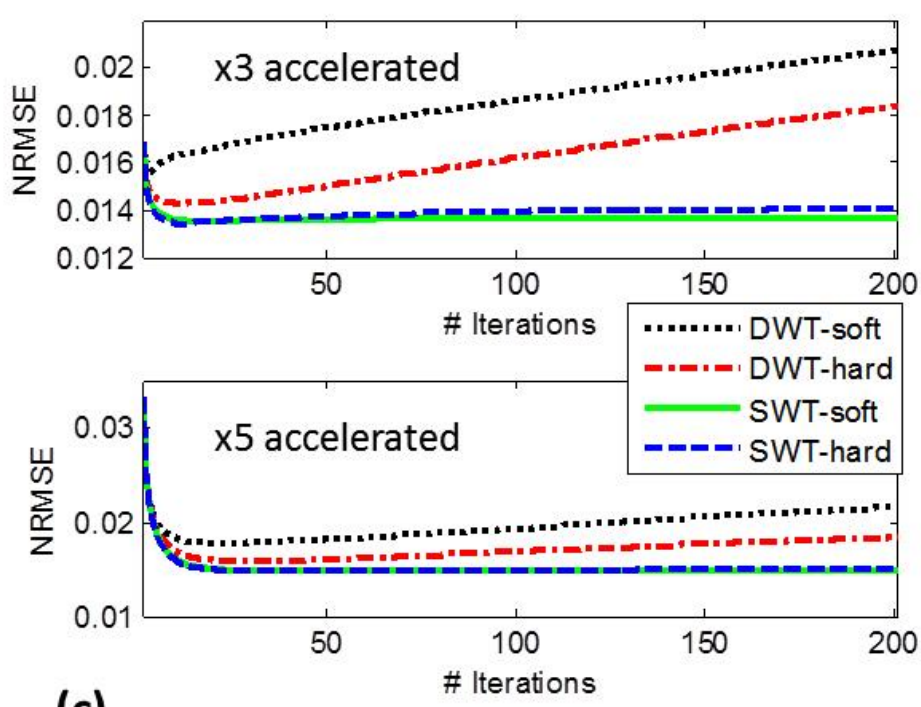

(c)
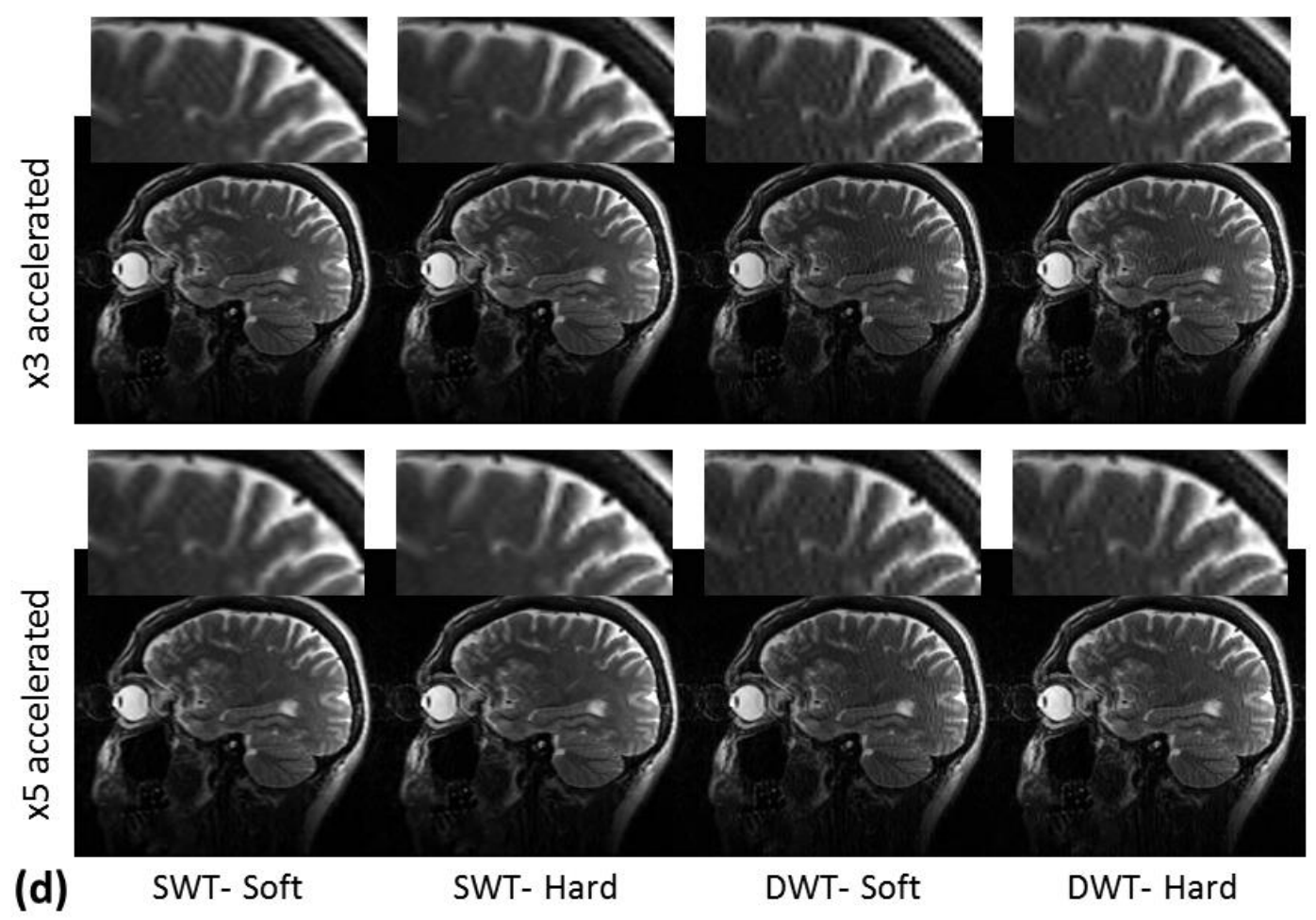

Figure 8- Reconstruction of under-sampled 32-channel FSE data by the multiple-coil iterative thresholding algorithm with SWT/DWT soft/hard thresholding. (a) Fully-sampled data. (b) Illustration of variable density random under-sampling. Note the fully-sampled center portion of k-space amounting to the auto-callibration data (c) Convergence plot of the multiple-coil iterative thresholding reconstruction algorithm, in terms of NRMSE vs. iteration number for $\mathrm{x} 3$ and $\mathrm{x} 5$ acceleration. (d) Examples of reconstruction from $\mathrm{x} 3$ and $\mathrm{x} 5$ under-sampled data after 50 iterations. Note the visual artifacts in the DWT reconstructions that are absent or greatly reduce in the corresponding SWT reconstructions. 
Figure 8 shows sample reconstructions by the multiple-coil iterative thresholding algorithm with SWT/DWT soft/hard thresholding. As illustrated in this figure, most of the artifacts in the DWT iterative soft/hard thresholding reconstructions are noticeably reduced in the corresponding SWT reconstructions.

The progress of the iterative reconstruction algorithms is shown in Figure 8(b). Not only do the SWT reconstructions result in lower reconstruction errors, the "overconvergence" effect ${ }^{6}$ in the DWT reconstructions, which results in an increase in the reconstruction error after a number of iterations before convergence, is not observed in the SWT reconstructions. This is more thoroughly investigated in Figure 9.

The dependence of the iterative SWT/DWT thresholding reconstructions on the choice of the threshold is illustrated in Figure 9. In the interest of space, only soft thresholding reconstructions are reported. Nevertheless, the main conclusions are applicable to hard thresholding also.

An initial base threshold was obtained using the Birgé-Massart strategy [26], in which the threshold is chosen such that at each decomposition level $j$, from 1 to $j_{m}$, $n_{j}$ largest decimated wavelet transform coefficients are kept, with $n_{j}=$ $M /\left(j_{m}+2-j\right)^{\alpha}$, where $M$ is typically assumed to be equal to the length of the coarsest approximation coefficients, and $\alpha=3$. The convergence of the iterative SWT/DWT thresholding algorithms, in terms of the reconstruction NRMSE vs. iteration number, was studied for several variations of this base threshold by multiplicative factors.

As shown in Figure 9, increasing the threshold generally resulted in increased reconstruction error for both the SWT and DWT reconstructions as well as increased over-convergence for the DWT reconstruction (dotted lines on the plots). On the other hand, a moderate decrease of the threshold did not result in noticeable improvement in the reconstruction error, nor did it alleviate the over-convergence observed with DWT, while a more aggressive decrease in the threshold resulted in increased reconstruction error due to increased over-convergence for both SWT and DWT. In general the results suggest that the Birgé -Massart strategy can be used to obtain practically optimum thresholds for both SWT and DWT.

Clearly, regardless of the threshold, SWT resulted in lower reconstruction errors compared to DWT. Additionally, the SWT reconstruction generally reached convergence in far less iterations than the corresponding DWT reconstruction, with no noticeable over-convergence. (For instance compare Figure 9(a) with Figure

\footnotetext{
${ }^{6}$ Over-convergence occurs when the optimum for the objective function being computed (in this case, the $l_{1}$ norm of the DWT coefficients) differs significantly from a desirable reference metric (such as the NRMSE between the reconstructed and fully-sampled images) often characterized by an initial sharp decrease in the reference metric followed by a more gradual increase.
} 
9(b,c): while SWT reaches convergence in about 50 iterations, it almost takes 5000 iterations for the DWT reconstruction to reach convergence.)

It should be noted that all the results in Figure 7, Table 3, and Figure 8 are obtained with thresholds obtained based on the Birgé -Massart strategy. Furthermore, in order to avoid giving SWT any advantage due to the over-convergence of the DWT reconstruction (see the discussion above on over-convergence), and since in practice the reconstructions can be terminated after a certain number of iterations, all the results in Figure 7, Table 3, and Figure 8(d) were obtained with 50 iterations.

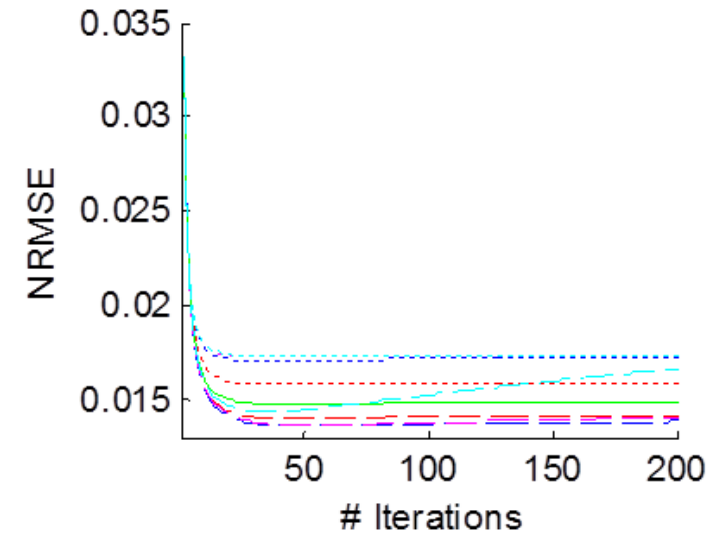

(a) SWT soft thresholding

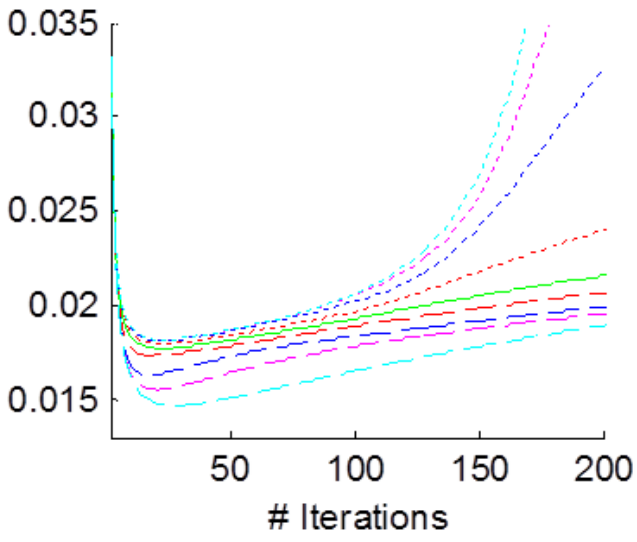

(b) DWT soft thresholding

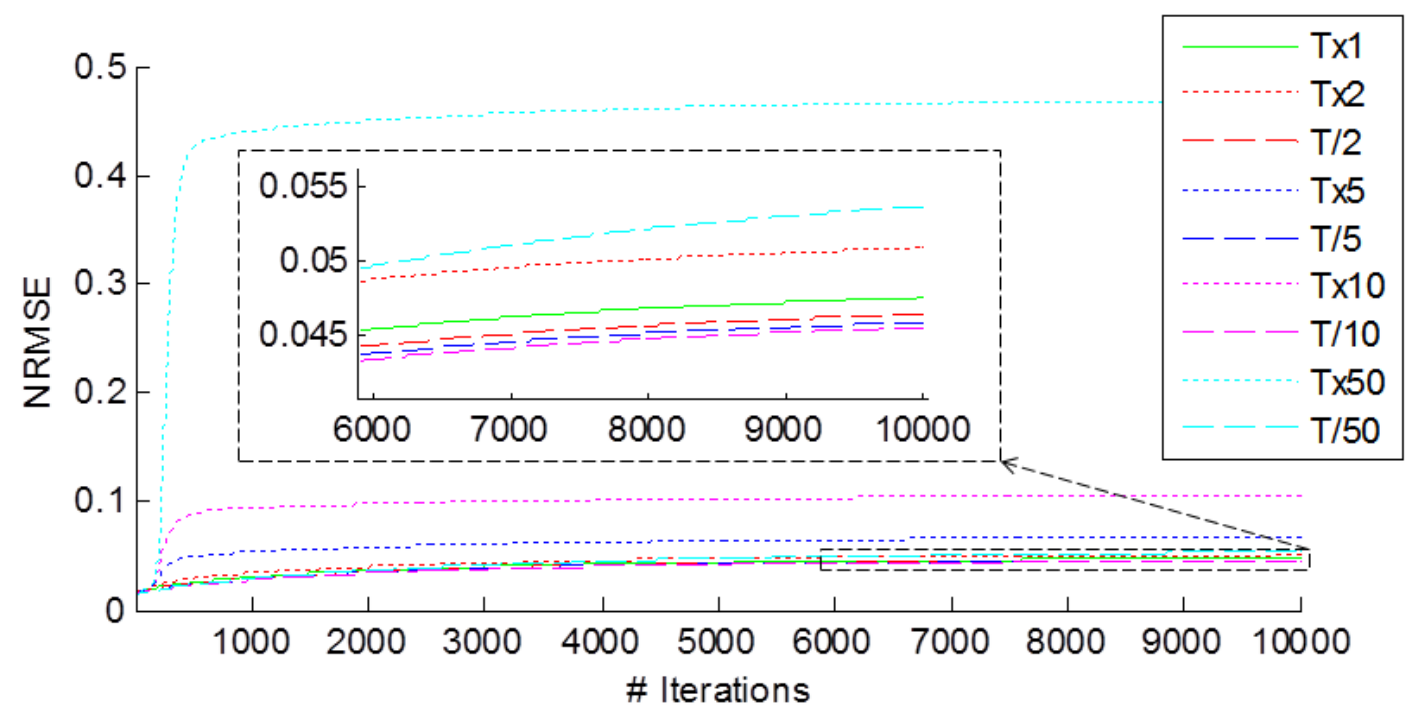

(c) DWT soft thresholding- extended

Figure 9- Effect of the choice of the threshold on the convergence of the multiple-coil iterative SWT/DWT soft thresholding algorithm for the reconstruction of under-sampled 32-channel FSE data (under-sampling factor 5) with SWT (a) and DWT (b, c). The convergence of the algorithms, in terms of the reconstruction NRMSE vs. iteration number, is shown for several variations of a base threshold, $T$, by multiplicative factors. Since the 
DWT reconstruction requires far more iterations to converge than the SWT reconstruction, an extended plot over 10000 iterations is shown in (c) for the DWT reconstruction.

Figure 10 shows the reconstruction performance of SWT/DWTRS/DWT SPIRiT on the same under-sampled 32-channel FSE datasets, in terms of the mean and the standard deviation of the reconstruction errors for the repeated trials. Clearly SWT results in lower mean error values than DWT and DWTRS. The mean and its 95\% confidence interval of the paired NRMSE differences are shown in Table 4. Similar to the previous experiments, negative NRMSE difference means with confidence intervals that do not include zero indicate that SWT resulted in lower reconstruction errors than DWT and DWTRS with all the findings showing significance after the Bonferroni correction.

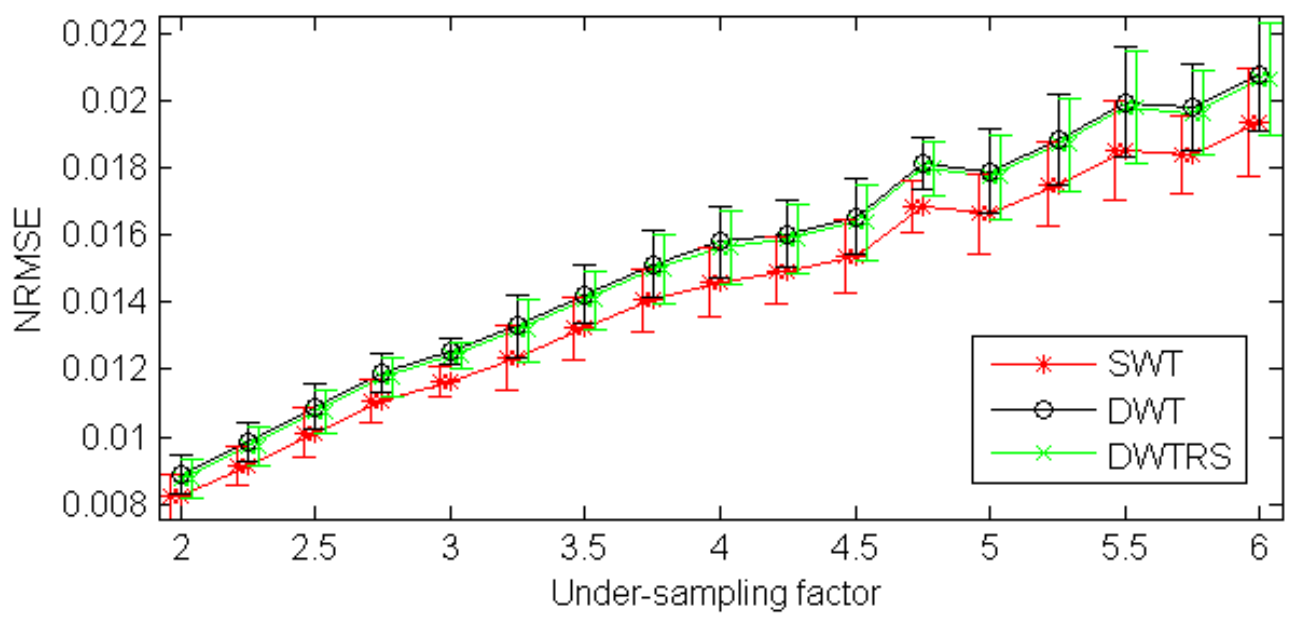

Figure 10- Mean NRMSE and the error bars of one standard deviation for the reconstruction of the under-sampled 32-channel FSE data by DWT/DWTRS/SWT SPIRiT.

\begin{tabular}{llllll}
$\left(\mathrm{x} 10^{-3}\right)$ & \multicolumn{7}{c}{} \\
\hline U.F. & $\mathbf{2}$ & $\mathbf{3}$ & $\mathbf{4}$ & $\mathbf{5}$ & $\mathbf{6}$ \\
\hline NRMSEswT - NRMSEDWT & $-0.67(8 \%)$ & $-0.91(8 \%)$ & $-1.2(8 \%)$ & $-1.3(7 \%)$ & $-1.4(7 \%)$ \\
& \pm 0.067 & \pm 0.082 & \pm 0.12 & \pm 0.10 & \pm 0.090 \\
NRMSEswT - NRMSEDWTRS & $-0.54(6 \%)$ & $-0.76(6 \%)$ & $-1.1(7 \%)$ & $-1.1(6 \%)$ & $-1.3(6 \%)$ \\
& \pm 0.066 & \pm 0.090 & \pm 0.13 & \pm 0.11 & \pm 0.095 \\
\hline
\end{tabular}

Table 4- Mean and its 95\% confidence interval of the paired NRMSE differences (i.e., NRMSE of the DWT/DWTRS reconstruction subtracted from that of the corresponding SWT reconstruction) for the reconstruction of the 32-channel FSE data by SWT/DWTRS/DWT SPIRiT for different under-sampling factors (U.F.). The numbers in brackets show the percentage of mean improvement with SWT over the corresponding DWT reconstruction. All findings are significant after the Bonferroni correction. 

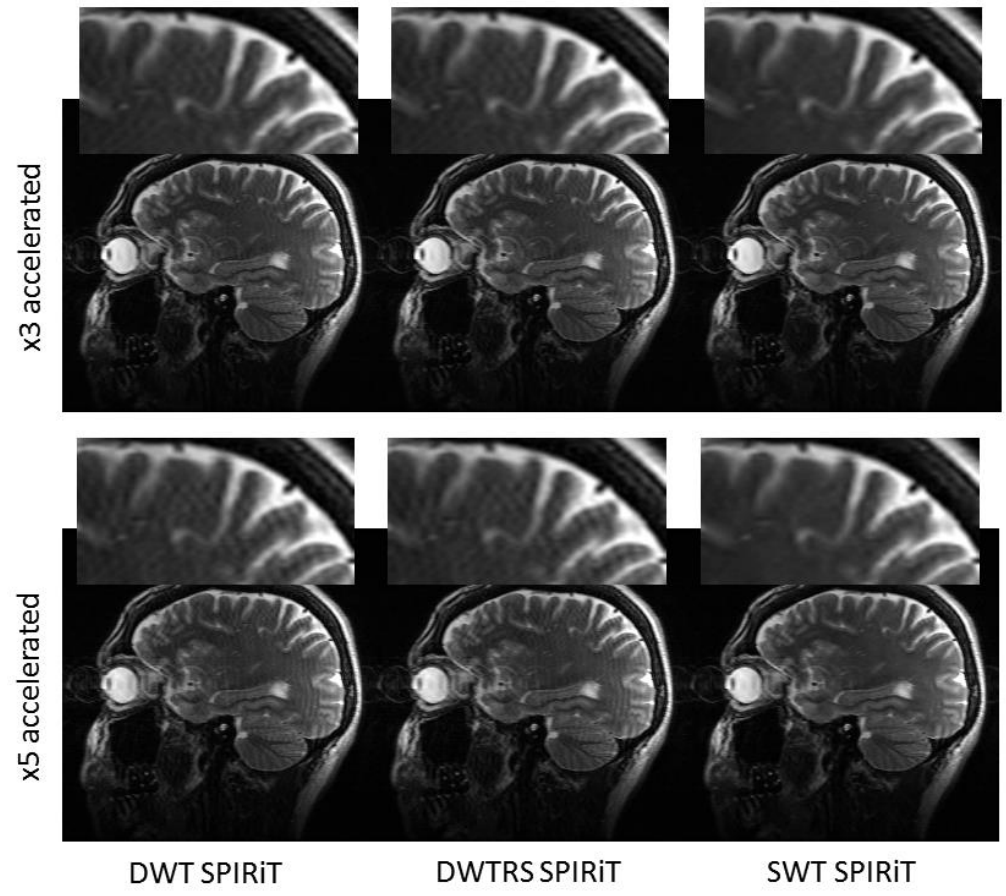

Figure 11-

Reconstruction of the under-sampled 32channel FSE data (under-sampling factor 3 and 5) by SWT/DWTRS/DWT SPIRiT. Note the visual artifacts in the DWT/DWTRS reconstructions that are absent or greatly reduced in the corresponding SWT reconstructions. Fully-sampled data are shown in Figure 8(a).

Sample reconstructions by SWT/DWTRS/DWT SPIRiT are shown in Figure 11. This figure clearly illustrates that most of the DWT (including DWTRS) reconstruction artifacts are absent or greatly reduced in the corresponding SWT reconstruction.

The progress of the SPIRiT reconstructions for various regularization parameters $(\lambda$ in equation 8 ) is shown in Figure 12. A base value $T$ for the regularization parameter was assumed as suggested in the code supplement to [9]. The convergence of the algorithm, in terms of the reconstruction NRMSE vs. iteration number, was studied for several variations of $T$ by multiplicative factors.

The convergence plots generally conform to those of the multiple-coil iterative thresholding algorithms in the sense that the SWT reconstructions results in lower reconstruction error and less over-convergence. Furthermore, as one may expect, DWTRS falls in between DWT and SWT both in terms of the reconstruction error and over-convergence.

All the results reported in Figure 10, Table 4, and Figure 11, are obtained with a regularization $T$ (corresponding to the green plot in Figure 12). Furthermore, in order to avoid giving the SWT reconstructions any advantage due to overconvergence, all the results in these figures and table are obtained with 100 iterations, i.e., around the minimum of the NRMSE curves for DWT and DWTRS. 

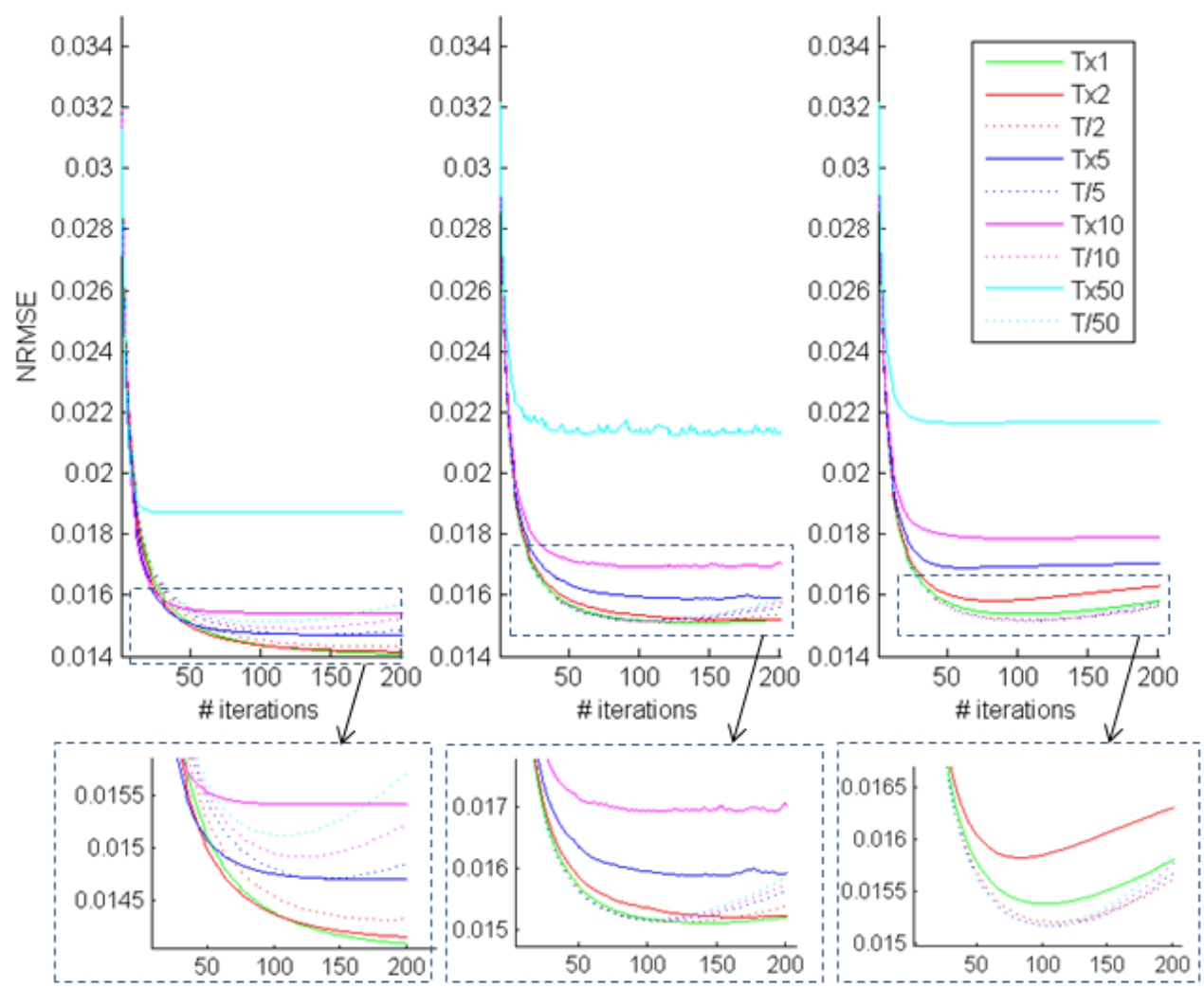

(a) SWT

(b) DWT-RS

(c) DWT

Figure 12- Effect of the choice of the regularization parameter on the convergence of the SPIRiT reconstruction algorithm for the reconstruction of under-sampled 32-channel FSE data (under-sampling factor 5) with several variations of the discrete wavelet transform, i.e., SWT, DWTRS, and DWT. The convergence of the algorithm, in terms of the reconstruction NRMSE vs. iteration number, is shown for several variations of a base regularization parameter, $T$, by multiplicative factors.

It is interesting to observe that the multiple-coil iterative SWT reconstructions and the SWT SPIRiT reconstructions result in similar reconstruction quality both visually and in terms of the reconstruction error, while the multiple-coil iterative DWT thresholding reconstructions suffer from more artifacts than the corresponding DWT SPIRiT reconstructions. This suggests that reasonable reconstructions can be achieved with simple [multiple-coil] iterative SWT thresholding, which is much less computational demanding than more complex algorithms such as SPIRiT. 
Furthermore, as noted previously, despite its redundancy, the non-decimated wavelet transform can be computed very efficiently- in $O(n \log (n))$ time. While it is still more computationally demanding compared with the decimated wavelet transform or its random-shifts version, which can be computed in $O(n)$, the visual and quantitative improvements are very noticeable. In addition, practical implementations show small execution time difference between SWT and DWT. For example, the execution time for the multiple-coil iterative thresholding reconstructions of Figure 8(d) were 8 and 9 seconds for DWT and SWT respectively, on an ordinary $3.40 \mathrm{GHz}$ PC using MATLAB.

Figure 13 shows sample reconstructions of the 3D SPGR data with 2D Poisson disk sampling. Clearly, also in this case, the SWT reconstructions result in noticeably less visual artifacts than the corresponding DWT reconstruction. The results are generally consistent with those of the 2D FSE data described above.
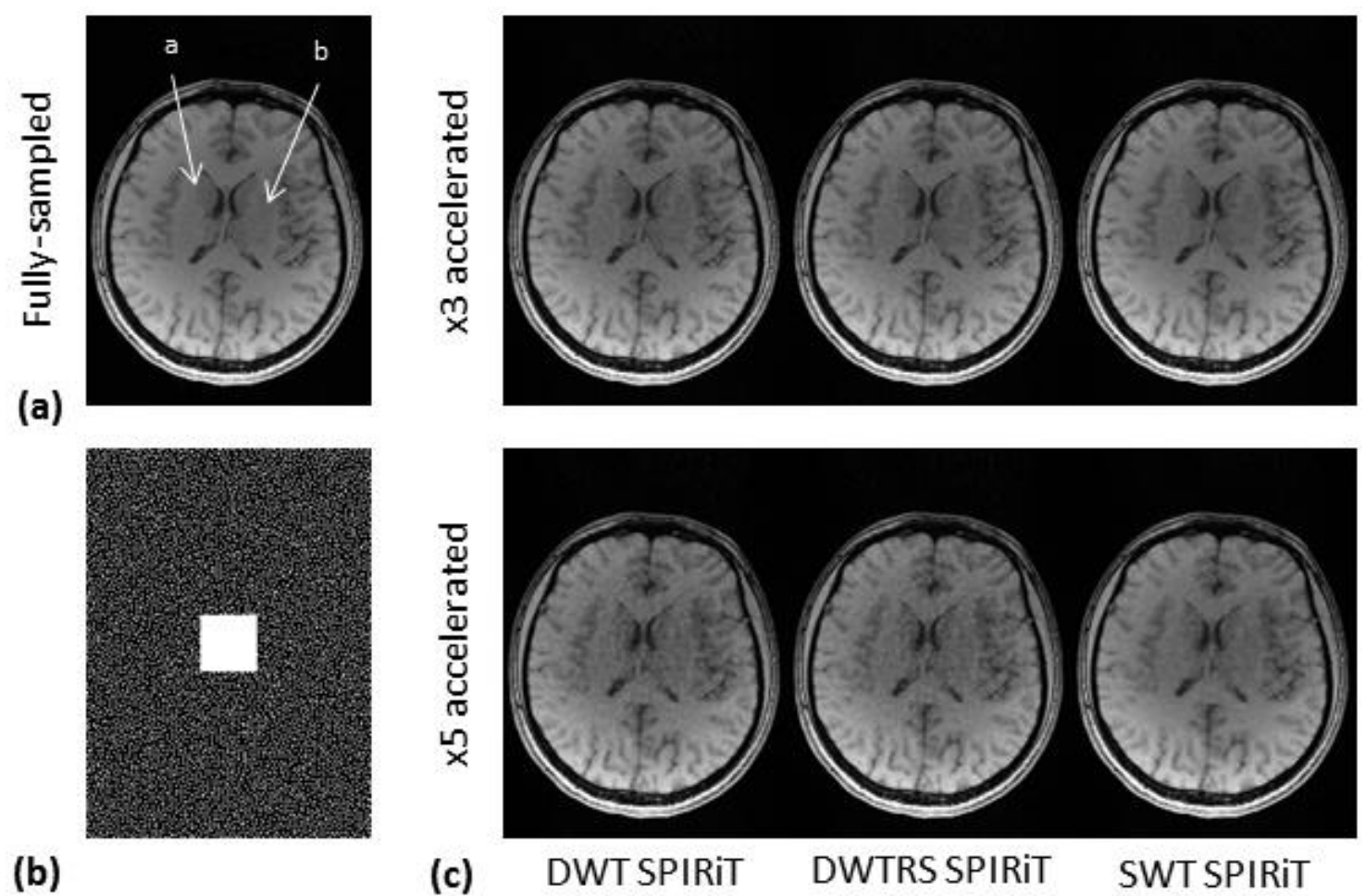

(b)

(c)

Figure 13- Reconstruction of 32-channel 3D SPGR data from x3 and x5 under-sampled data by SWT/DWTRS/DWT SPIRiT. (a) Fully-sampled data. (b) illustration of Possion disk under-sampling. Note the fully-sampled center portion of k-space amounting to the autocallibration data. (c) examples of reconstructions by SPIRiT with DWT, DWT with random shifts (DWTRS), and SWT from x3 and x5 under-sampled data. Note the reduced artifacts with SWT reconstructions. For example, compare the visibility of the head of caudate (pointed to by arrow a in the fully-sampled image) and the putamen (pointed to by arrow $\mathrm{b}$ in the fully-sampled image) in the images. In particular, note that while these structures are are barely visible in the DWT and DWTRS reconstructions at $\mathrm{x} 5$ acceleration due to heavy artifacts, they are more clearly visible in the corresponding SWT reconstruction. 


\section{Discussion and conclusion}

The most important conclusion drawn from the results presented in this article is that under-sampled MRI reconstructions based on the stationary wavelet transform (SWT) exhibit noticeably fewer visual artifacts than the corresponding decimated wavelet transform (DWT) reconstructions.

While quantitative quality measures, e.g., the normalized root mean square error (NRMSE), are commonly used to measure the reconstruction performance, these quantities do not necessarily provide a good measure of the practical quality perceived by radiologists and other expert users of these medical images. In fact, it was called to authors' attention by collaborating radiologists and neurosurgeons that images with a very high quantitative reconstruction quality may still suffer from potentially critical losses that those quantitative measures fail to capture. This issue is being more thoroughly investigated in the ongoing work, in which we are investigating the relationship between the quantitative quality measures and the perceptual quality scores, as given by radiologists and other expert users, for different reconstructions and applications.

Nevertheless, the results also indicate that SWT reconstructions result in approximately $10-30 \%$ improvement in the reconstruction error compared to the corresponding DWT reconstructions for the reconstruction of multi-channel data. This improvement is statistically significant, and is robust to the particular reconstruction algorithm chosen. Additionally, SWT results in faster convergence than DWT. Also, the over-convergence effect in the DWT reconstruction, where the reconstruction error reaches its minimum before convergence and increases thereafter, is not observed with SWT. These concepts were demonstrated with different pulse sequences, $\mathrm{k}$-space sampling, and reconstruction approaches.

\section{References}

[1] A. Macovski, "Noise in MRI," Magn. Reson. Med., vol. 36, no. 3, pp. 494-497, 1996.

[2] K. P. Pruessmann, M. Weiger, M. B. Scheidegger, and P. Boesiger, "SENSE: Sensitivity encoding for fast MRI," Magn. Reson. Med., vol. 42, no. 5, pp. $952-$ 962, 1999.

[3] M. A. Griswold, P. M. Jakob, R. M. Heidemann, M. Nittka, V. Jellus, J. Wang, B. Kiefer, and A. Haase, "Generalized autocalibrating partially parallel acquisitions (GRAPPA)," Magn. Reson. Med., vol. 47, no. 6, pp. 1202-1210, 2002.

[4] D. K. Sodickson and W. J. Manning, "Simultaneous acquisition of spatial harmonics (SMASH): Fast imaging with radiofrequency coil arrays," Magn. Reson. Med., vol. 38, no. 4, pp. 591-603, 1997. 
[5] E. J. Candes, J. Romberg, and T. Tao, "Robust uncertainty principles: exact signal reconstruction from highly incomplete frequency information," Inf. Theory IEEE Trans. On, vol. 52, no. 2, pp. 489-509, 2006.

[6] E. J. Candes and M. B. Wakin, "An Introduction To Compressive Sampling," Signal Process. Mag. IEEE, vol. 25, no. 2, pp. 21-30, 2008.

[7] M. Lustig, D. Donoho, and J. M. Pauly, "Sparse MRI: The application of compressed sensing for rapid MR imaging," Magn. Reson. Med., vol. 58, no. 6, pp. 1182-1195, Dec. 2007.

[8] D. L. Donoho, "Compressed sensing," IEEE Trans. Inf. Theory, vol. 52, no. 4, pp. 1289 -1306, Apr. 2006.

[9] M. Lustig and J. M. Pauly, "SPIRiT: Iterative self-consistent parallel imaging reconstruction from arbitrary k-space," Magn. Reson. Med., vol. 64, no. 2, pp. 457-471, 2010.

[10] I. Daubechies, M. Defrise, and C. De Mol, "An iterative thresholding algorithm for linear inverse problems with a sparsity constraint," Commun. Pure Appl. Math., vol. 57, no. 11, pp. 1413-1457, Nov. 2004.

[11] R. R. Coifman and D. L. Donoho, "Translation-invariant de-noising," Wavelets Stat. Springer Lect. Notes Stat, vol. 103, pp. 125-150, 1995.

[12] J. Pesquet, H. Krim, and H. Carfantan, "Time-invariant orthonormal wavelet representations," IEEE Trans. Signal Process., vol. 44, no. 8, pp. 1964-1970, 1996.

[13] G. P. Nason and B. W. Silverman, "The Stationary Wavelet Transform and some Statistical Applications," in Wavelets and Statistics, A. Antoniadis and G. Oppenheim, Eds. Springer New York, 1995, pp. 281-299.

[14] M. A. T. Figueiredo and R. D. Nowak, "An EM algorithm for wavelet-based image restoration," IEEE Trans. Image Process., vol. 12, no. 8, pp. 906-916, 2003.

[15] M. Guerquin-Kern, M. Haberlin, K. P. Pruessmann, and M. Unser, "A Fast Wavelet-Based Reconstruction Method for Magnetic Resonance Imaging," IEEE Trans. Med. Imaging, vol. 30, no. 9, pp. 1649-1660, 2011.

[16] S. S. Vasanawala, M. J. Murphy, M. T. Alley, P. Lai, K. Keutzer, J. M. Pauly, and M. Lustig, "Practical parallel imaging compressed sensing MRI: Summary of two years of experience in accelerating body MRI of pediatric patients," in Biomedical Imaging: From Nano to Macro, 2011 IEEE International Symposium on, 2011, pp. $1039-1043$.

[17] I. W. Selesnick, R. G. Baraniuk, and N. C. Kingsbury, "The dual-tree complex wavelet transform," IEEE Signal Process. Mag., vol. 22, no. 6, pp. 123-151, 2005.

[18] M. N. Do and M. Vetterli, "The contourlet transform: an efficient directional multiresolution image representation," IEEE Trans. Image Process., vol. 14, no. 12, pp. 2091-2106, 2005.

[19] E. J. Candès and D. L. Donoho, Curvelets - A Surprisingly Effective Nonadaptive Representation For Objects with Edges. 2000.

[20] Yookyung Kim, Maria I. Altbach, Theodore P. Trouard, and Ali Bilgin, "Compressed sensing using dual-tree complex wavelet transform," in International Society for Magnetic Resonance in Medicine, 2009, vol. 17, p. 2814. 
[21] Z. Zhu, K. Wahid, P. Babyn, and R. Yang, “Compressed Sensing-based MRI Reconstruction Using Complex Double-density Dual-tree DWT," J. Biomed. Imaging, vol. 2013, pp. 10:10-10:10, Jan. 2013.

[22] X. Qu, W. Zhang, D. Guo, C. Cai, S. Cai, and Z. Chen, "Iterative thresholding compressed sensing MRI based on contourlet transform," Inverse Probl. Sci. Eng., vol. 18, no. 6, pp. 737-758, 2010.

[23] J. Ma, "Improved Iterative Curvelet Thresholding for Compressed Sensing and Measurement," IEEE Trans. Instrum. Meas., vol. 60, no. 1, pp. 126-136, 2011.

[24] M. Murphy, M. Alley, J. Demmel, K. Keutzer, S. Vasanawala, and M. Lustig, "Fast SPIRiT Compressed Sensing Parallel Imaging MRI: Scalable Parallel Implementation and Clinically Feasible Runtime," IEEE Trans. Med. Imaging, vol. 31, no. 6, pp. 1250-1262, 2012.

[25] S. D. Sharma, C. L. Fong, B. S. Tzung, M. Law, and K. S. Nayak, “Clinical Image Quality Assessment of Accelerated Magnetic Resonance Neuroimaging Using Compressed Sensing," Invest. Radiol., Mar. 2013.

[26] L. Birgé and P. Massart, "From Model Selection to Adaptive Estimation," in Festschrift for Lucien Le Cam, D. Pollard, E. Torgersen, and G. L. Yang, Eds. Springer New York, 1997, pp. 55-87.

[27] Y. Tsaig and D. L. Donoho, "Extensions of compressed sensing," Signal Process., vol. 86, no. 3, pp. 549-571, Mar. 2006.

[28] A. A. Samsonov, E. G. Kholmovski, D. L. Parker, and C. R. Johnson, "POCSENSE: POCS-based reconstruction for sensitivity encoded magnetic resonance imaging," Magn. Reson. Med., vol. 52, no. 6, pp. 1397-1406, 2004.

[29] P. B. Roemer, W. A. Edelstein, C. E. Hayes, S. P. Souza, and O. M. Mueller, "The NMR phased array," Magn. Reson. Med. Off. J. Soc. Magn. Reson. Med. Soc. Magn. Reson. Med., vol. 16, no. 2, pp. 192-225, Nov. 1990.

[30] D. J. Larkman and R. G. Nunes, "Parallel magnetic resonance imaging," Phys. Med. Biol., vol. 52, no. 7, pp. R15-R55, Apr. 2007. 